\title{
Fine Structure of Vegetative Hyphae of Rhizopus
}

\author{
By LILIAN E. HAWKER aNd PATRICIA McV. ABBOTT \\ Department of Botany, University of Bristol
}

(Received 16 June 1962)

\begin{abstract}
SUMMARY
Ultrathin sections of vegetative hyphae of Rhizopus sexualis and $R$. homothallicus examined by electron microscopy revealed structure essentially similar to that reported for some other filamentous fungi and yeasts. The cell wall in section consists of elongated elements tangentially orientated. The nucleus is surrounded by a double membrane interrupted by pores and contains a denser body identified as the nucleolus. The cytoplasm contains well-defined mitochondria, vesicles (cisternae), unidentified spherical bodies, oil drops and vacuoles. There is a complex system of cytoplasmic membranes or membrane-like layers including the outer layer or plasmalemma, membranes (tonoplasts) surrounding the vacuoles and, in the zone of extension growth behind the hyphal tip, a more or less continuous, convoluted 'cortical membrane' separating a central core of cytoplasm from a peripheral zone. The possible significance of such a membrane in relation to cytoplasmic streaming is discussed.
\end{abstract}

\section{INTRODUCTION}

The physiology of zygospore formation in Rhizopus sexualis (Smith) Callen and some other members of the Mucorales is being studied in this laboratory (Hawker, Hepden \& Perkins, 1957; Hepden \& Folkes, 1960; Hepden \& Hawker, 1961; Hawker \& Hepden, 1963). Unpublished observations with the light microscope suggest that chemical changes occur in the hyphae before conjugation. A study of the fine structure of the hyphae, as revealed by the electron microscope, was therefore made to assist interpreting experimental results. Additional observations have been made with another homothallic species, Rhizopus homothallicus Hesseltine \& Ellis (Hesseltine \& Ellis, 1961) and with a single strain of Rhizopus nigricans Ehrenb.

\section{METHODS}

Rhizopus sexualis was grown on $3 \%$ malt agar for $24 \mathrm{hr}$. at $20^{\circ} ; \boldsymbol{R}$. homothallicus for $30 \mathrm{hr}$. at $25^{\circ}$. Tufts of young hyphae were picked off from the edge of a colony and fixed immediately. A single tuft of hyphae from an older culture of R. homothallicus was also fixed.

The methods of fixation, dehydration and embedding outlined below were based on information supplied by Dr E. H. Mercer of the Chester Beatty Institute, and on a recent publication (Mercer \& Birbeck, 1961).

Fixation. The hyphae were either (a) fixed in Luft's permanganate, $2 \%(\mathrm{pH} 7)$ (Luft, 1956) in a refrigerator for $20 \mathrm{~min}$., centrifuged for $10 \mathrm{~min}$. and then washed in cold distilled water, or $(b)$ fixed by a dual fixation method described by Thyagarajan, 
Conti \& Naylor (1961) as follows; Luft's permanganate diluted to $1.5 \%$ with buffer ( $\mathrm{pH}$ adjusted to $\mathbf{7 \cdot 0}$ ), for $10 \mathrm{~min}$. at room temperature, washed with cold distilled water, followed by fixation in osmium tetroxide, $1.0 \%(\mathrm{pH} \mathrm{7 \cdot 2})$ for $70 \mathrm{~min}$. in a refrigerator, centrifuged for $10 \mathrm{~min}$. while still in the osmic fixative and washed with veronal buffer.

Dehydration. After fixation the hyphae were left for $15 \mathrm{~min}$. in 30, 50 and $80 \%$ alcohol successively and were finally left for $1 \mathrm{hr}$. in absolute alcohol.

Embedding. Surplus alcohol was drained off and the tuft of hyphae gently dried with filter paper and placed in a 50/50 mixture ( $/ v / v)$ of alcohol and 'Araldite' (a proprietary epoxide embedding medium) for $1 \mathrm{hr}$. The hyphae were then passed through two changes of araldite mixture (without accelerator) and left in the second of these overnight. They were finally placed in gelatine capsules containing araldite to which accelerator had been added, and were left at $62^{\circ}$ for $2-3$ days until the araldite had hardened.

Sectioning. Sectioning proved to be unusually difficult owing to the exceptionally tough walls of the hyphae. Sections of these fungi were cut on a Huxley ultramicrotome (Cambridge Instrument Co.). A diamond knife proved unsatisfactory and a new glass knife was used for every set of sections. The microtome was usually set to cut sections $0.05 \mu$ thick, but the actual thickness probably varied from 0.05 to $0 \cdot 1 \mu$. A few thicker sections $(0 \cdot 15 \mu)$ of Rhizopus homothallicus were cut.

The sections were floated on $10 \%$ acetone and caught on small copper grids of the usual type. Owing probably to the tough nature of the cell wall it was difficult to get properly stretched thin sections as the araldite tended to tear away from the sections of hyphae.

Electron microscopy. The sections were examined and photographed with a Phillips E.M. 200 or occasionally a Phillips E.M. 100 electron microscope in the Department of Physics, University of Bristol.

\section{RESULTS}

The main object of the investigation was to determine the structure of the cytoplasm of vegetative hyphae in young cultures before the onset of reproduction. Observations were also made of the structure of the nucleus and cell wall.

\section{The cell wall}

The cell wall was seen only in section. It consists of elongated elements laid down tangentially, giving it a striate appearance (Pl. 2, fig. 8). The wall varies in thickness from 0.05 to $0.5 \mu$. It is assumed that thickness is correlated with distance from the hyphal tip, so affording a means of estimating the relative physiological age of the hyphae. There is no visible difference in structure between the outer older portions and the inner newly formed layers of the thick wall of a mature hypha. Wall structure was similar in both species of Rhizopus studied.

\section{Cell contents}

Nuclei. The nuclei are relatively large bodies (c. 1·25-2.2 $\mu$ diam.), approximately globose or ellipsoidal but showing minor irregularities in outline (Pl. 1, figs. 1 , 4; Pl. 2, fig. 5). 
The internal mass of the nuclei shows no clearly defined structure. There is usually a denser patch which, by comparison with suitably stained preparations viewed under the light microscope, is assumed to be the nucleolus ( $\mathrm{Pl}$. 1, fig. 1; Pl. 2, fig. 5).

The nuclei are surrounded by a clearly defined double membrane interrupted by pores (Pl. 2, fig. 5). Occasionally the nuclear membrane is seen to be in direct continuity with membranous inclusions in the cytoplasm. The nuclear substance was in direct contact with the cytoplasm through pores in the nuclear membrane.

Although the presence of paired nuclei suggests that nuclear division occurs fairly frequently, there is no indication that discrete chromosomes are formed. Robinow (1957), from a study of stained material of Mucor hiemalis, M. fragilis and Phycomyces blakesleeanus by the light microscope, concluded that 'division of the chromatinic elements is direct and involves neither spindle nor metaphase plate.'

Mitochondria. Numerous clearly defined mitochondria are present in hyphae of all ages. In transverse sections they are usually more or less circular or broadly elliptical (Pl. 1, figs. 1, 2; Pl. 2, figs. 5, 7) but in longitudinal sections of young hyphae many of them are elongated (Pl. 1, fig. 4; Pl. 2, fig. 6). They are surrounded by a double membrane, the inner layer of which is infolded to form a varying number of cristae. In relatively thick sections these are seen to be lamellae and are frequently arranged in groups of two or more roughly parallel plates. In young hyphae there are relatively few cristae and they extend less deeply into the central matrix of the mitochondria than do those of the mitochondria of older hyphae. The range of size of the mitochondria is similar in all parts of the hyphae (i.e. 0.4-0.7 $\mu \times 0.5-0.8 \mu$ ).

Intact hyphae stained with toluidine blue and examined under the light microscope show numerous mitochondria (Pl. 2, figs. 11, 12) and confirm the electron microscope findings relating to size, shape and distribution.

Vesicles, tubules or cisternae; possible Golgi bodies. The cytoplasm also contains sparsely distributed irregular vesicles or tubules (Pl. 1, figs. 2, 3; Pl. 2, figs. 6, 7) resembling the cisternae seen by Blondel \& Turian (1960) in Allomyces. Occasionally these are grouped together but are not so regular in arrangement as the typical Golgi apparatus seen in plant cells by Buvat (1958) and others.

Unidentified spherical bodies. In some sections there are groups of irregularly arranged spherical bodies with homogeneous contents. These did not stain black with osmium fixation and their nature was not determined.

Oil drops and food vacuoles. Numerous homogeneous globose bodies, stained black in material fixed with osmic acid, are thought to be oil drops. They occur as clear white patches of regular outline in material fixed with permanganate only (Pl. 2, fig. 7). Material stained with Sudan III and examined under the light microscope contains oil drops of similar size and distribution.

Stellate vacuoles (Manton, 1961) containing amorphous material are also present (Pl. 2, fig. 7). They resemble the storage vacuoles in conidiophores of Stilbum zacoloxanthum McAlear \& Edwards (1959). The nature of the stored material is not known, but suitably stained hyphae under the light microscope show the presence of considerable quantities of glycogen.

Cytoplasmic 'membranes'. A thin dark layer, the plasmalemma, appearing as a membrane consisting of one or more layers, can be seen enclosing the cytoplasm. Similar layers (tonoplasts) surround the vacuoles. The plasmalemma is not always 
in close contact with the wall and may show a crinkled edge (Pl. 2, fig. 8). The curious structures termed lomasomes by Moore \& McAlear (1961) and thought by them to be characteristic of fungi, were not seen, although occasionally vacuoles could be seen in contact with the plasmalemma and apparently about to break through it (Fig. 1). Large vacuoles, present only in mature hyphae, have a regular outline (Fig. 2) but smaller ones in younger hyphae have irregular or stellate shape (Pl. 2, fig. 7). All are surrounded by a distinct 'membrane'. Often one vacuole is invaginated by another, suggesting that the surrounding layer is a definite membrane.

In addition to the plasmalemma and the membranes surrounding the vacuoles there is a complex system of membrane-like layers in the endoplasm. These are most clearly marked in sections which, from the extreme thinness of the cell wall, must have been taken at or near the tip of the hypha, probably from the zone of extension growth just behind it. In many such sections (both transverse and longitudinal) a complete or almost complete sinuous 'cortical membrane' could be traced, separating an irregular central core from a peripheral zone (Pl. 1, figs. 1-4; Fig. 3).

The core is always less dense than the peripheral layer and contains all or most of the nuclei and few mitochondria. The peripheral zone is often crowded with mitochondria (Fig. 3). The presence of such zoning is confirmed by observation of living hyphae by phase contrast (Pl. 2, figs. 9, 10).

In slightly older hyphae, as judged by the rather thicker cell walls, the 'cortical membrane' is no longer continuous but is broken and tends to envelop small stellate vacuoles (Pl. 2, fig. 7). In still older hyphae, with relatively thick walls, the small vacuoles coalesce and may extend across the central part of the hyphae, leaving only a shallow peripheral zone of cytoplasm containing nuclei and numerous mitochondria, neither of which show signs of deterioration (Fig. 2). The vacuoles of hyphae at this stage still show contents thought to be food reserves, probably glycogen. In very old hyphae the vacuole occupies a still greater volume and no longer contains regularly spaced material but is apparently empty or contains irregular masses of unidentifiable substances. The peripheral cytoplasmic residues in such hyphae are irregular and mitochondria and nuclei are distorted. They remain recognizable, however, even after the endoplasm shows signs of disintegration.

\section{DISCUSSION}

No complete study of fine structure in a member of the Mucorales has yet been published, although the structure of the wall of Phycomyces blakesleeanus has been investigated (Roelofsen, 1959) and a single electron micrograph of a section of a hypha of a species of Syncephalastrum has been published by Moore \& McAlear (1961). A few fungi from other groups have been studied, notably Allomyces macrogynus (Blondel \& Turian, 1960), Neurospora crassa (Shatkin \& Tatum, 1959) and various yeasts (Thyagarajan et al. 1961; Kawakami \& Nehira, 1959). The structure of the hyphae of Rhizopus sexualis differs from that of other fungi studied, chiefly in the greater development of 'membranes' in the cytoplasm of the young hyphae. This may not be a real difference, since other studies may not have included such young hyphae. 


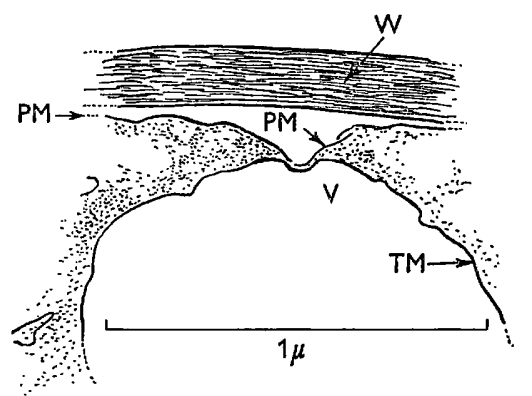

Fig. 1

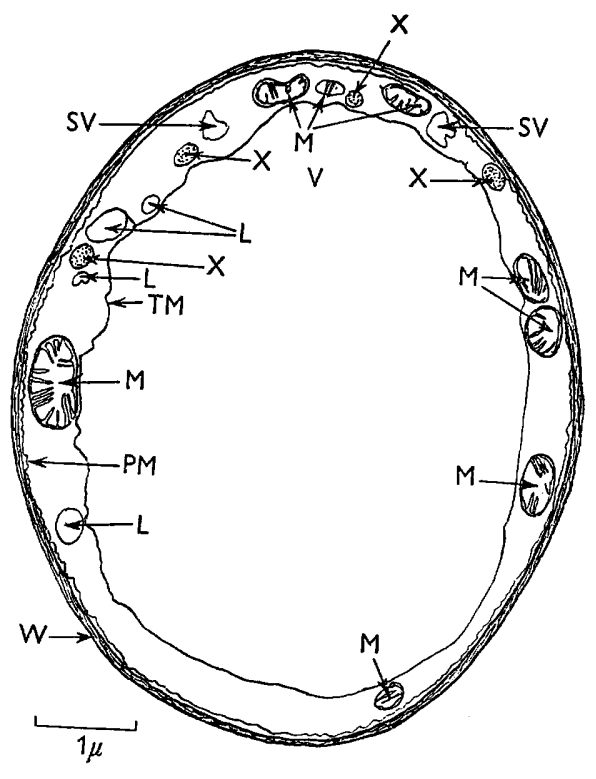

Fig. 2

Fig. 1. Rhizopus homothallicus, tracing from electron micrograph showing vacuole (V) in contact with plasmalemma (PM) and apparently about to break through it. $W=$ cell wall, TM = tonoplast.

Fig. 2. Rhizopus homothallicus, tracing of electron micrograph of T.S. of mature hypha. Note large central vacuole (V) and peripheral cytoplasm. $\mathbf{M}=$ mitochondria, $\mathbf{P M}=$ plasmalemma, $\mathbf{T M}=$ tonoplast, $\mathrm{W}=$ cell wall, $\mathbf{L}=$ lipid body, $\mathbf{S V}=$ small stellate vacuole, $\mathrm{X}=$ spherical body of unknown nature.

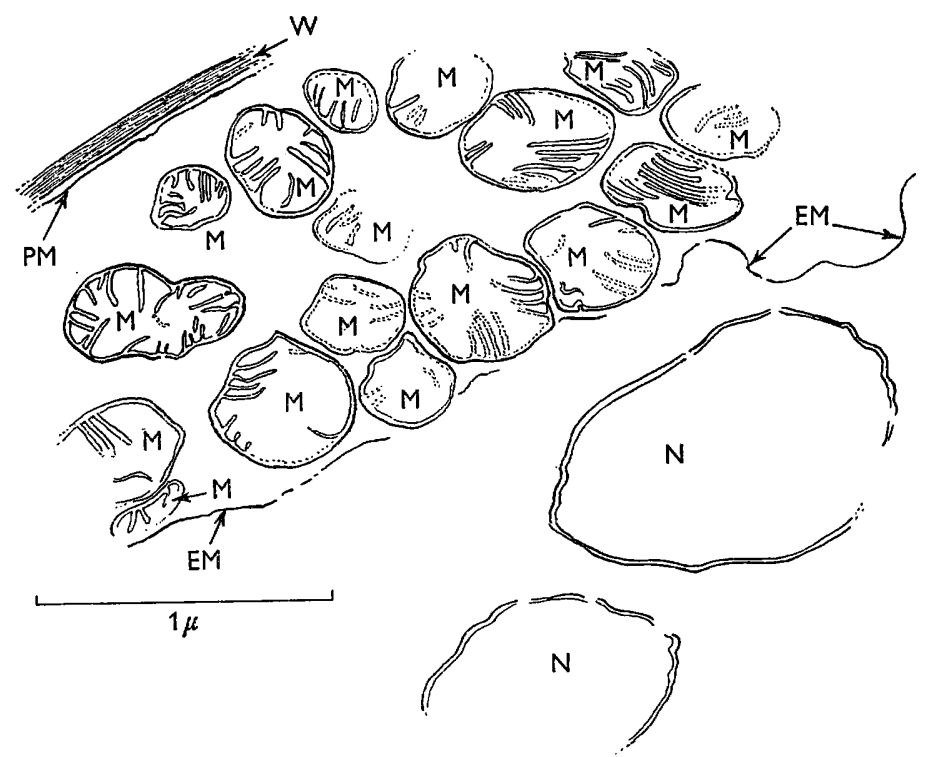

Fig. 3. Rhizopus sexualis, tracing of electron micrograph showing endoplasmic cortical membrane separating outer peripheral zone containing numerous mitochondria from inner central cylinder containing nuclei. $\mathbf{M}=$ mitochondria, $\mathbf{N}=$ nucleus, $\mathbf{P M}=$ plasmalemma, $\mathbf{E M}=$ endoplasmic membrane, $\mathbf{W}=$ cell wall. 
The general structure of the nuclei, mitochondria and other cytoplasmic inclusions resembles that described for other fungi.

The most interesting feature in the anatomy of Rhizopus is the separation of an outer zone containing numerous mitochondria from an inner core containing nuclei and a few mitochondria in the region of extension growth of the hypha. These zones appear to be separated by a membrane, often of two or even three layers. It is not certain whether this is a true membrane or merely the effect of two surfaces in contact. Such a division is of particular interest in view of the observation of Arthur (1897) with Rhizopus nigricans, confirmed by Schröter (1905) and Buller (1933), of the occasional presence of particles moving in a direction opposite to that of the main stream within the hyphae. These authors describe a broad vacuolated axial stream of protoplasm and a sheath-like peripheral return current of nonvacuolated protoplasm moving basipetally. This observation has been doubted by some later workers and it has been suggested that such a streaming of protoplasm in opposite directions in one and the same hypha at the same time presupposes the existence of a cytoplasmic membrane separating the two streams of cytoplasm. We examined living hyphae under the ordinary light microscope and by phase contrast. In mature parts of the living hypha streaming was rapid, but at any particular time was in one direction only. In the region of extension growth near the apex of the hyphae, however, streaming was usually slower but some particles could be seen moving towards the tip while others were moving away from it. The particles were of a size comparable with that of mitochondria. The peripheral particles moved in jerks as if they were encountering obstacles. Plate 1, figs. 1-4, indicate that such obstacles may well exist in the form of folds of the 'cortical membrane'. The great concentration of mitochondria in the peripheral zone suggests that these may be actively engaged in the linear extension of the cell wall. The internal arrangement of this part of the hyphae and the observations on particle movement are consistent with the hypothesis that food material and nuclei move up the centre of the young hypha and that mitochondria may accumulate at the tip and then move slowly down the peripheral layer. Their progress is impeded by folds of the membrane and it may be that during this slow backward progress they are concerned in the addition of new material to the growing wall. Buller (1933) reported the presence of small moving particles in the peripheral stream. In older parts of the hypha, where the membranes are not continuous, the cytoplasmic streaming is unidirectional at any particular time. Particles flowing in this stream together with small vacuoles are apparently unimpeded by any resistant structure, in contrast to those moving nearer the tip. This is again consistent with the observed structure of older hyphae in which continuous cortical membranes are lacking.

We wish to thank Dr J. Fraymouth of this Department for advice on methods of fixing, embedding and sectioning the material and Mr G. A. Bassett and Mrs J. Dyer of the Physics Department, University of Bristol, for taking many of the electron micrographs. We also wish to thank Dr C. W. Hesseltine of the U.S. Department of Agriculture, Peoria, Illinois for the culture of Rhizopus homothallicus.

The work was made possible by a grant from the Department of Scientific and Industrial Research, to whom our grateful thanks are due. 


\section{REFERENCES}

Arthur, J. C. (1897). The movement of protoplasm in coenocytic hyphae. Ann. Bot., Lond. 11, 491.

Blondel, B. \& Turian, G. (1960). Relation between basophilia and fine structure of cytoplasm in the fungus Allomyces macrogynus $\mathrm{Em}$. J. biophys. biochem. Cytol. 7, 127.

Buller, A. H. (1933). Researches on Fungi, Vol. 5, London: Longmans.

Buvat, R. (1958). Nouvelles observations sur l'appareil de Golgi dans les cellules des végétaux vasculaires. C.R. Acad. Sci., Paris, 246, 2157.

Hawker, L. E., Hepden, P. M. \& Perkins, S. M. (1957). The inhibitory effect of low temperature on early stages of zygospore production in Rhizopus sexualis. J. gen. Microbiol. $17,758$.

HAwKen, L. E. \& HePden, P. M. (1963). Sporulation in Rhizopus sexualis and some other fungi following a period of intense respiration. Ann. Bot., Lond., N.S. (in the Press).

HePden, P. M. \& Folkes, B. F. (1960). A possible relationship between nucleic acid metabolism and the initiation of zygospores of Rhizopus sexualis. Nature, Lond. 185, 254.

Hepden, P. M. \& Hawker, L. E. (1961). A volatile substance controlling early stages of zygospore formation in Rhizopus sexualis. J. gen. Microbiol. 24, 155.

Hesseltine, C. W. \& Ellis, J. J. (1961). Notes on Mucorales, especially Absidia. Mycologia, 53, 406.

KaWAKami, N. \& NeHIRA, T. (1959). Electron-microscopy of fungi. IX. Intracellular structures of Rhodotorula glutinis and Nadsonia fulvescens and their relation to the physiological characters and taxonomic affinity. J. form. Tech. 37, 125. (Japanese with English summary.)

LuFT, J. H. (1956). Permanganate-a new fixative for electron microscopy. J. biophys. biochem. Cytol. 2, 799.

Manton, I. (1961). Plant cell structure. In Contemporary Botanical Thought, p. 171. Eds. A. M. McLeod and L. S. Cobley. Edinburgh: Oliver \& Boyd.

McAlear, J. H. \& EDwards, G. A. (1959). Continuity of plasma membrane and nuclear membrane. Exp. Cell. Res. 16, 689.

Mercer, E. H. \& Birbeck, M. S. C. (1961). Electron Microscopy. Oxford: Blackwells.

Moore, R. T. \& McAlear, J. H. (1961). Fine structure of Mycota. 5. Lomasomespreviously uncharacterized hyphal structures. Mycologia, 53, 194-200.

RoBinow, C. F. (1957). The structure and behaviour of the nuclei in spores and growing hyphae of the Mucorales. I. Mucor hiemalis and Mucor fragilis. II. Phycomyces blakesleeanus. Canad. J. Microbiol. $3,771$.

Roelorsen, P. A. (1959). The plant cell wall. Plates 62 and 65. Handbuch der Pflanzenanatomie, 3. Berlin: Gebrüder Borntraeger.

Schröter, A. (1905). Über Protoplasmaströmung bei Mucorineen. Flora, 45, 1.

Shatkin, A. J. \& Tatum, E. L. (1959). Electron microscopy of Neurospora crassa mycelia. J. biophys. biochem. Cytol. 6, 423.

Thyagarajan, T. R., Conti, S. F. \& Naylor, H. B. (1961). Electron microscopy of yeast mitochondria. Exp. Cell Res. 25, 216.

\section{EXPLANATION OF PLATES}

Rhizopus-electron micrographs

$\mathbf{W}=$ cell wall, $\mathbf{N}=$ nucleus, $\mathbf{N U}=$ nucleolus, $\mathbf{N M}=$ nuclear membrane, $\mathbf{N P}=$ pores in nuclear membrane, $\mathbf{M}=$ mitochondria, $\mathbf{M C}=$ cristae, $\mathbf{C}=$ cisternae, $\mathbf{P M}=$ plasmalemma, TM = tonoplast, $\mathbf{E M}=$ endoplasmic or 'cortical' membranes, $\mathbf{S V}=$ stellate vacuole, $\mathbf{L}=$ lipid or oil drop, $\mathrm{X}=$ unidentified spherical bodies. Figs. 1-4, fixed permanganate and osmium tetroxide; figs. 5-8, fixed permanganate only.

Plate 1. Rhizopus sexualis

Fig. 1. T.S. hypha through zone of extension growth behind hyphal tip, as indicated by extremely thin cell wall. Note complex endoplasmic membrane enclosing a central 'core' containing a nucleus; peripheral zone is denser and contains numerous mitochondria and cisternae. 
Figs. 2, 3. Parts of same section (outlined by dotted lines in fig. 1) photographed at higher magnification. Fig. 2 shows thin wall of tangentially arranged elements, thin plasmalemma indicated by arrow, mitochondria and lobed endoplasmic 'membranes'. Fig. 3 shows similar endoplasmic membranes of complex nature, small endoplasmic vesicles or cisternae and an unidentified spherical body.

Fig. 4. A longitudinal tangential section of a similar hypha, showing central zone containing a nucleus and small vacuoles and a peripheral zone containing mitochondria and vacuoles. Mitochondria, vacuoles and nucleus are longitudinally elongated and orientated in direction of cytoplasmic streaming.

Plate 2. Rhizopus homothallicus, R. nigricans and R. sexualis

Figs. 5, 6. T.S. and L.S. respectively of young hypha of $R$. homothallicus, showing nucleus with nucleolus, 3-layer nuclear membrane interrupted by pores; mitochondria with complex membrane, inner layer of which is infolded to form plate-like cristae arranged parallel to one another in groups and extending well into the interior; endoplasm with sparsely distributed endoplasmic reticulum, which in fig. 5 shows continuity with nuclear membrane; small vacuoles; unidentified spherical bodies; plasmalemma and thin cell wall. Note elongated mitochondria in fig. 6 .

Fig. 7. T.S. older hypha of $\boldsymbol{R}$. homothallicus showing mitochondria, vacuoles, lipid bodies, sparse endoplasmic reticulum with occasional cisternae, plasmalemma and cell wall.

Fig. 8. Section through cell wall of old hypha of $\boldsymbol{R}$. homothallicus showing tangentially striate structure.

Figs. 9. 10. Hypha of $R$. nigricans vitally stained with Janus green, photographed at two different focusings under phase contrast. Note roughly cylindrical central column in zone of extension growth, indicating presence of 'cortical' membrane or the juxtaposition of masses of cytoplasm of different texture.

Figs. 11, 12. Young hyphae of $R$. sexualis, stained with toluidine blue, photographed under oil immersion lens, showing mitochondria and nuclei (clear bodies with stained nucleolus, one nucleus outlined with dots). Note elongated shape of mitochondria in older hypha. 


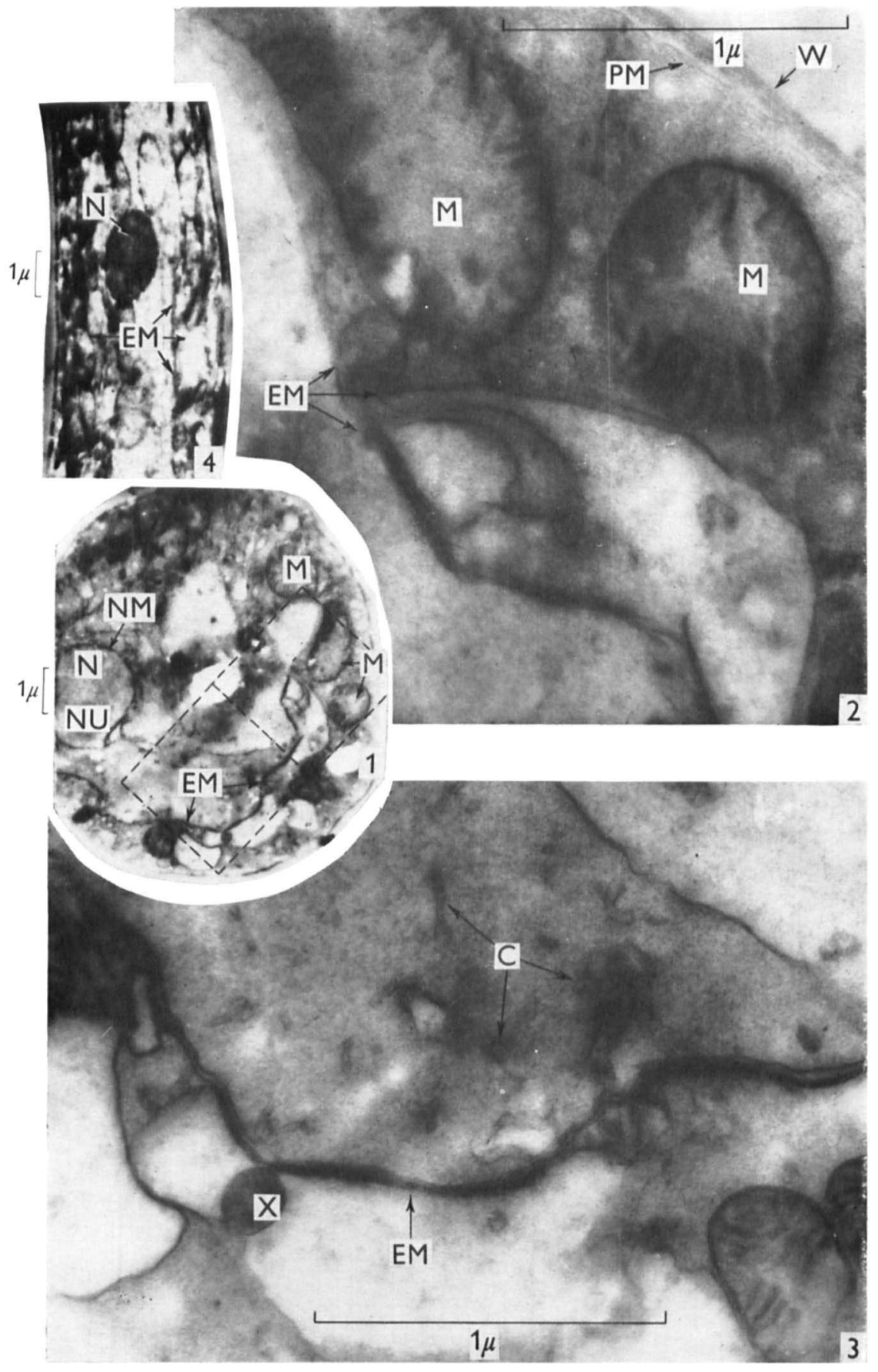




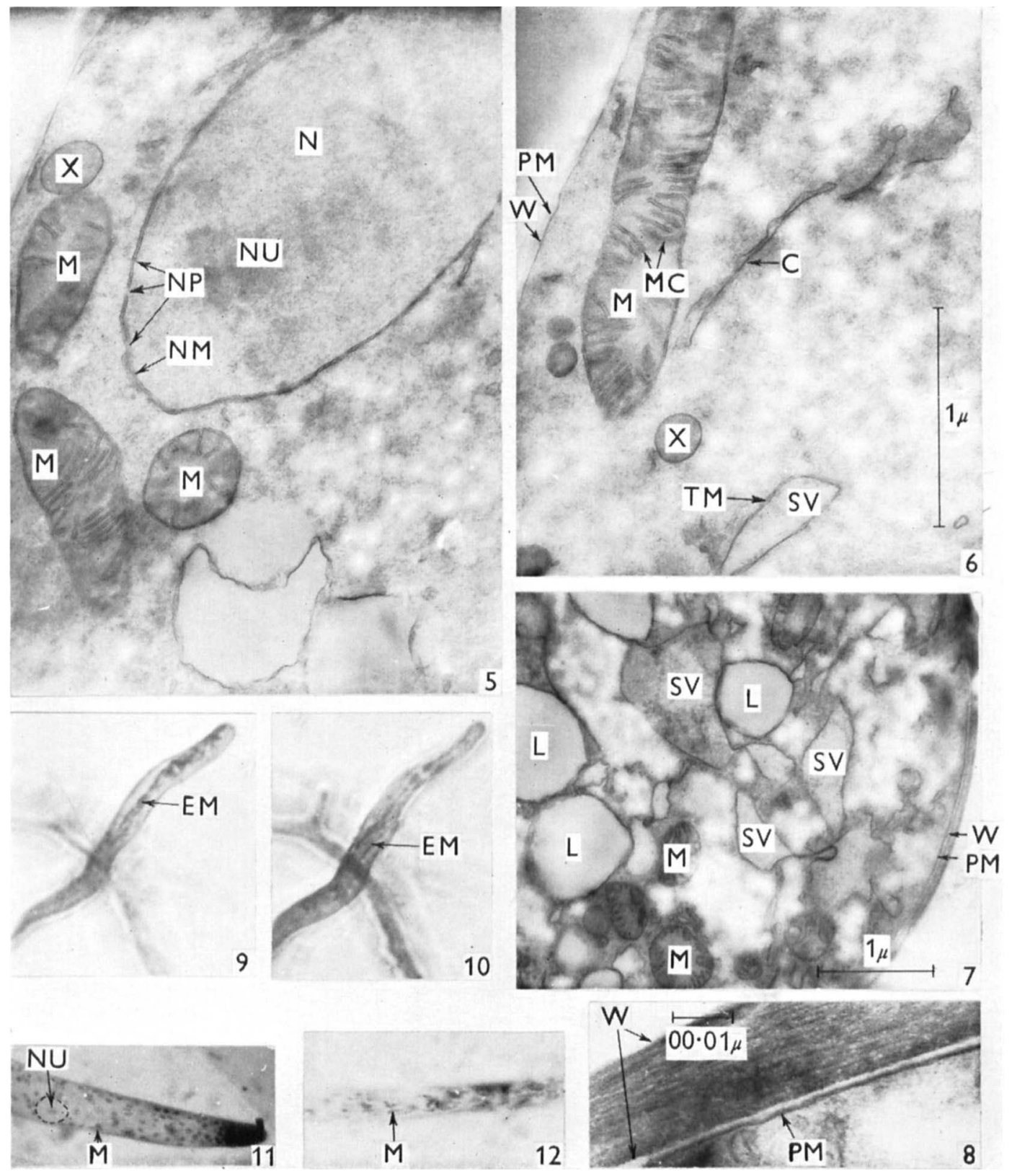

